\title{
Study of Yttria-Tantala Binary Using Scanning Transmission Electron Microscopy
}

\author{
Daesung Park ${ }^{1}$, Chandra Macauley ${ }^{1}$, Abel Fernandez ${ }^{1}$ and Carlos Levi ${ }^{1}$ \\ ${ }^{1}$ Materials Department, University of California, Santa Barbara, CA 93106-5050, United States
}

$\mathrm{ZrO}_{2}$ stabilized by $\mathrm{Y}^{3+}$ and $\mathrm{Ta}^{5+}$ has attracted much attention of scientists in the field of high temperature materials due to high toughness resulting from ferroelastic toughening and low thermal conductivity [1]. Other compositions in $\mathrm{ZrO}_{2}-\mathrm{YO}_{1.5}-\mathrm{TaO}_{2.5}$, such as $\mathrm{Y}_{2} \mathrm{O}_{3}$ rich fluorite and $\mathrm{YTaO}_{4}$ exhibited reliable mitigation of molten silicate and lower thermal conductivity compared to $\mathrm{ZrO}_{2}$ rich tetragonal phase, respectively.

While $\mathrm{ZrO}_{2}-\mathrm{YO}_{1.5}$ binary has been studied extensively, very few previous studies of the $\mathrm{YO}_{1.5}-\mathrm{TaO}_{2.5}$ binary can be found $[2,3]$. In this study, to elucidate plausible phases and provide fundamental understanding of phase transformation in the $\mathrm{YO}_{1.5}-\mathrm{TaO}_{2.5}$ binary, most important compositions were selected and characterized in detail. $\mathrm{Y}_{3} \mathrm{TaO}_{7}(75 \mathrm{Y} 25 \mathrm{Ta}(\mathrm{mol} \%))$ and $\mathrm{Y}_{7} \mathrm{Ta}_{3} \mathrm{O}_{18}(72 \mathrm{Y} 28 \mathrm{Ta}(\mathrm{mol} \%))$ compositions were synthesized via reverse co-precipitation of precursor solution and uniaxially compressed into pellets $10 \mathrm{~mm}$ in diameter. The pellets were heat treated at $1500^{\circ} \mathrm{C}$ and $1600^{\circ} \mathrm{C}$ for 100 hours.

The chemical composition of the bulk samples were measured by wavelength dispersive spectroscopy (WDS) and for local chemistry analysis energy dispersive X-ray (EDX) spectroscopy in transmission electron microscope (TEM) was performed. In addition, crystal structures of all samples were investigated by X-ray diffraction (XRD) and Raman spectroscopy. To provide a deep insight into crystal structure and phase evolution in local area electron diffraction analysis in TEM was performed. Furthermore, atomic resolution high-angle annular-dark field (HAADF) imaging was applied to show detail information of cation orderings due to the benefit of Z-contrast. In order to improve the signal-tonoise ratio while reducing scan and specimen drifts, 30 fast-scan images were sequentially recorded, aligned using cross-correlation algorithm and averaged out.

$72 \mathrm{Y} 28 \mathrm{Ta}$ heat treated at $1500^{\circ} \mathrm{C}$ for 100 hours showed a domain structure within the grains showing strong stripes in bright field image. In some domains coherent twin boundaries were observed and the stripes observed in the domain were identified as antiphase boundary proven by atomic resolution HAADF images. Similarly domain structures, twin and antiphase boundaries were observed in the $75 \mathrm{Y} 25 \mathrm{Ta}$ specimen annealed at $1500^{\circ} \mathrm{C}$ for 100 hours as shown in the atomic resolution HAADF image (Figure 1). But, the size of domains was smaller compared to $72 \mathrm{Y} 28 \mathrm{Ta}$ annealed at $1500^{\circ} \mathrm{C}$. In the $75 \mathrm{YTa} 25$ specimen annealed at $1600^{\circ} \mathrm{C}$ for 100 hours, ultrathin multilayer structure was observed showing repeated orthorhombic $(\mathrm{O})$ and fluorite $(\mathrm{F})$ cation ordering as shown in Figure 2(a). The $\mathrm{O}$ and $\mathrm{F}$ cation orderings are exhibited with atomic model overlaid in the HAADF image. To verify those cation orderings based on Z-contrast of the HAADF image, the position of every atomic column in Figure 2(a) was determined by fitting to two dimensional Gaussian function and their intensities were measured within a small disk with a finite radius. The average intensity profile along the in-plane direction as a function of atomic layer is shown in Figure 2(b). The average intensity profile proves the ultrathin multilayer structure of $\mathrm{O}$ and $\mathrm{F}$ cation ordering, suggesting partial phase transformation of $75 \mathrm{Y} 25 \mathrm{Ta}$ at $1600^{\circ} \mathrm{C}$ showing a good agreement with the previous study [3]. 


\section{References:}

[1] Kim, D.-J., Journal of the American Ceramic Society 73 (1990), p. 115-120.

[2] I. A. Bondar, A.I. Kalinin, and L.N. Koroleva, Inorganic Materials 8 (1973), p. 1649-1650.

[3] Y. Yokogawa and M.Yoshimura, Journal of the American Ceramic Society 74 (1991), p 2077-2081.

[4] The authors acknowledge funding from the NSF-GOALI program, DMR-1105672.

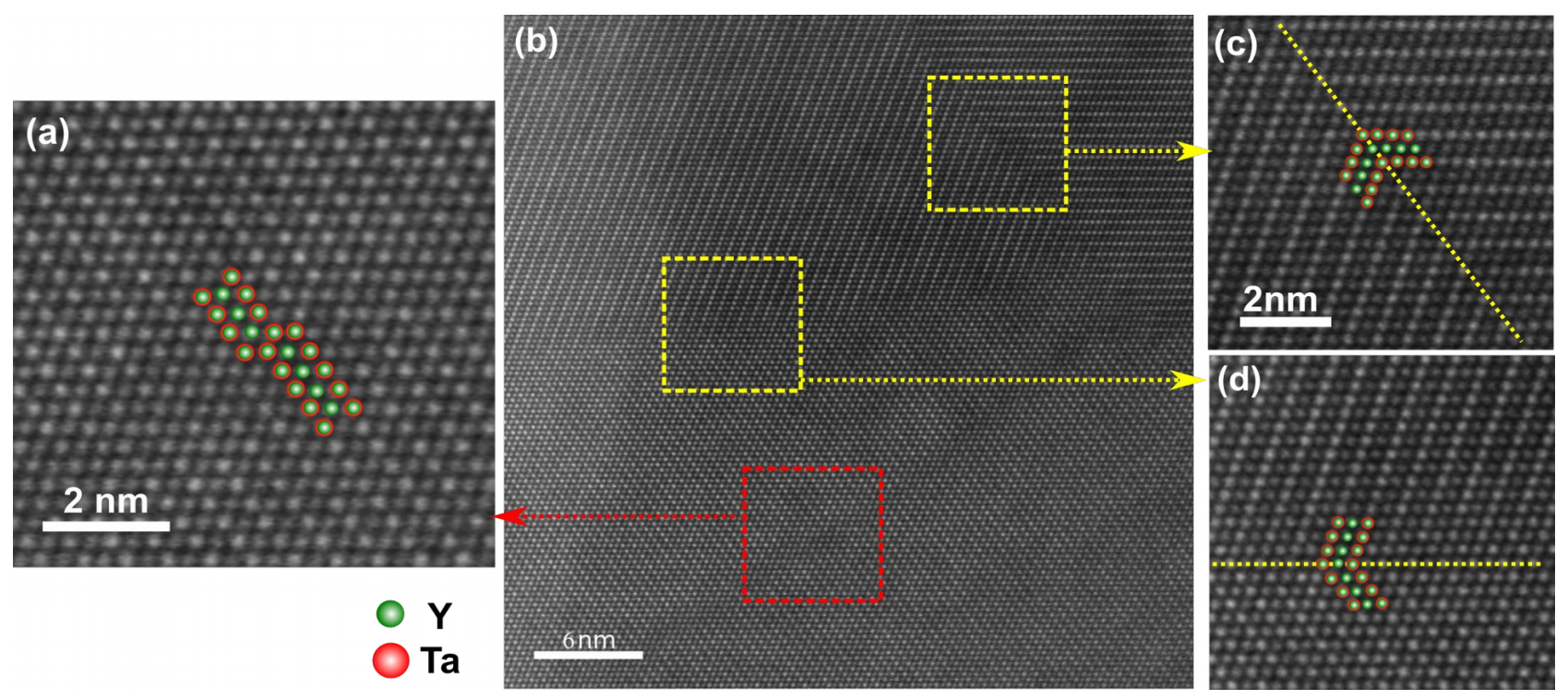

Figure 1. HAADF image of the $75 \mathrm{Y} 25 \mathrm{Ta}$ specimen $\left(1500^{\circ} \mathrm{C}\right.$ for 100 hours $)$ at the domain boundaries (b), antiphase boundary along the [010] zone axis (a) and twin boundaries along the [112] zone axis in (c) and (d).

(a)

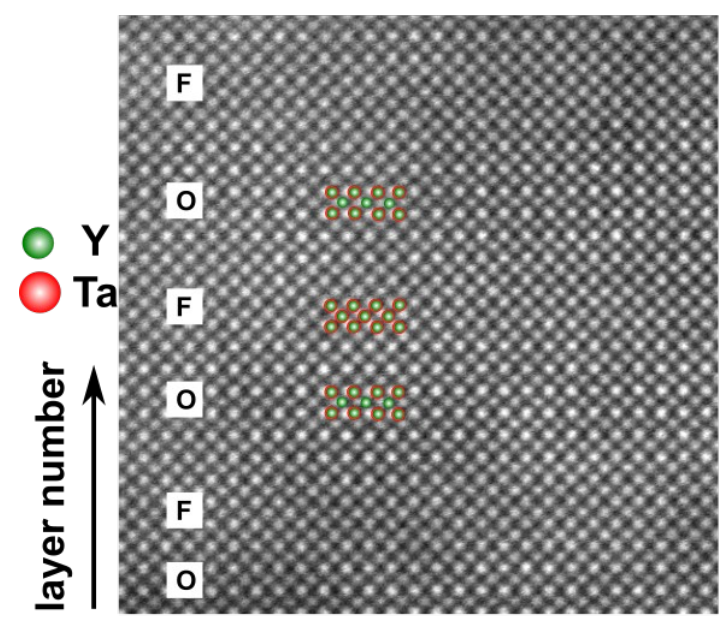

(b)

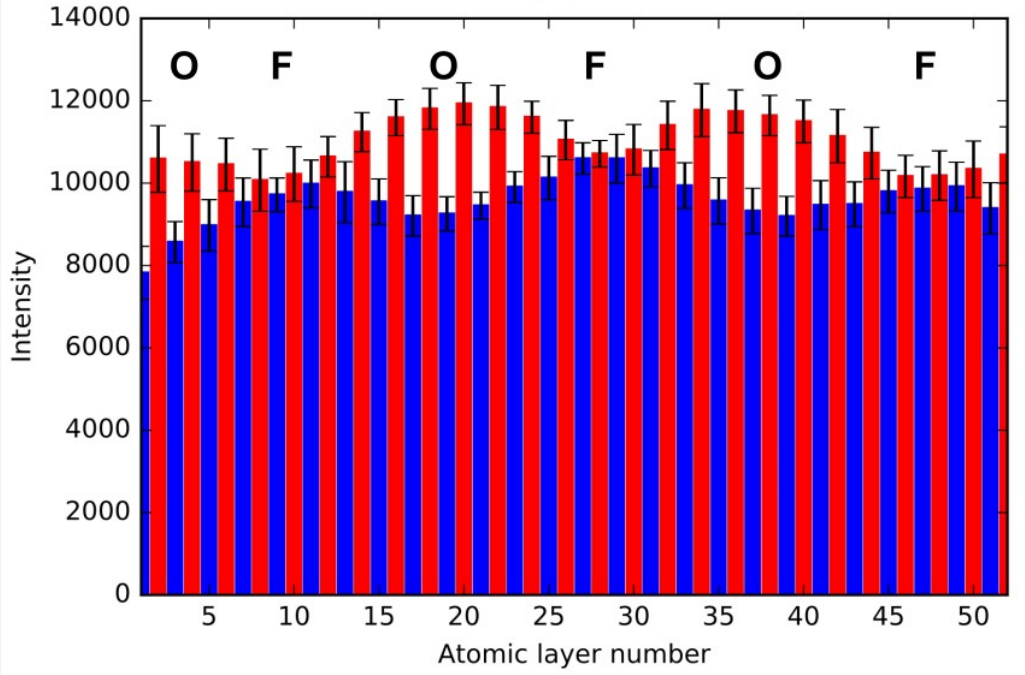

Figure 2. HAADF image along the [100] direction of the $75 \mathrm{Y} 25 \mathrm{Ta}$ specimen heat treated at $1600^{\circ} \mathrm{C}$ for 100 hours (a). Average intensity profile along the in-plane direction as a function of atomic layer (b). Multilayer structure of orthorhombic $(\mathrm{O})$ and fluorite $(\mathrm{F})$ cation ordering was clearly proved. 